\title{
PROPOSTA DI UNA FUNZIONE UTILITÀ DIPENDENTE DALL'UTILITÀ ATTESA E DALLA DISPERSIONE DELLE UTILITÀ
}

\author{
Nota del s.c. VITTORIO B. FROSINI (*)
}

(Adunanza del 20 ottobre 2011)

\begin{abstract}
SuNTO. - L'autore presenta le proprietà e le implicazioni di una proposta concernente una particolare sintesi statistica delle utilità contenute in una data prospettiva probabilistica. Seguendo un suggerimento di Maurice Allais, tale statistica è crescente con l'utilità attesa, e decrescente - per la maggior parte degli individui, che sono avversi al rischio - con la deviazione media assoluta delle utilità; l'introduzione di un parametro, che moltiplica tale misura di dispersione, permette di caratterizzare i comportamenti avversi oppure favorevoli al rischio, per mezzo del suo segno e del suo valore assoluto. Viene dimostrato che tale statistica (a) soddisfa la condizione di dominanza stocastica, (b) soddisfa la condizione di indipendenza, (c) soddisfa il "problema della assicurazione probabilistica", (d) risolve i paradossi di Allais, Ellsberg e Kahneman-Tversky (paradosso dell'assioma di sostituzione), (e) la deviazione media assoluta non può essere sostituita dallo scarto quadratico medio (o deviazione standard).
\end{abstract}

$$
* * *
$$

ABSTRACT. - The author develops the properties and implications of a proposal, concerning a summary statistic of the random prospect of utilities. Following a suggestion of Maurice Allais, such a statistic is increasing with expected utility, and decreasing for most people, who are risk averse - with the mean absolute deviation of utilities; a parameter multiplying this dispersion measure allows for risk averse or risk prone behaviour, according to its sign, and also for more or less departure from a certain

(*) Università Cattolica del Sacro Cuore, Milano, Dipartimento di Scienze statistiche. E-mail: benito.frosini@unicatt.it 
prospect. It is demonstrated that this statistic (a) satisfies the first stochastic dominance, (b) satisfies the independence condition, (c) satisfies the so called "problem of probabilistic insurance", (d) resolves the paradoxes of Allais, Ellsberg and Kahneman-Tversky (paradox of the substitution axiom), (e) the mean absolute deviation from the mean cannot be replaced by the standard deviation.

\section{INTRODUZIONE. IL CRITERIO DELL'UTILITÀ ATTESA E LA DISPERSIONE DELLE UTILITÀ}

Questa memoria è un contributo alla modellazione del comportamento individuale riguardo alla scelta fra più prospettive probabilistiche; tali prospettive probabilistiche sono definite a partire da prospettive certe, come di seguito definite.

Un individuo è posto di fronte alla scelta fra due o più prospettive, che coinvolgono prospettive certe $b_{1}, h_{2}, \ldots, b_{n}$, fra le quali è in grado di stabilire un ordinamento di preferenza. ( $b \leq k$ se $b$ è non preferito oppure è equivalente a $k$ ); le prospettive sono probabilistiche nel senso che l'individuo deve scegliere fra più prospettive del tipo

$d=\left[h_{1}, p_{1} ; h_{2}, p_{2} ; \ldots ; h_{n}, p_{n}\right]$

nelle quali cioè $b_{1}$ può accadere con probabilità $p_{1}, h_{2}$ può accadere con probabilità $p_{2}$ ecc. $\left(\sum p_{i}=1\right)$. In molte applicazioni, per semplicità, le prospettive certe $h_{i}$ sono ridotte a somme di danaro; tali prospettive ricevono anche il nome di lotterie. Le probabilità in discorso possono essere probabilità oggettive, ma è presumibile che siano spesso probabilità soggettive, cioè dipendenti dalla valutazione che ne viene fatta dal particolare individuo, cui è demandata la decisione di scelta fra due o più prospettive probabilistiche.

Vi è accordo generale che le prospettive certe $b_{1}, \ldots, h_{n}$ entrano nella valutazione e nel confronto fra le stesse prospettive non direttamente, ma attraverso la loro utilità per l'individuo; la prospettiva $d$ è perciò sostituita dalla prospettiva

$d=\left[u\left(b_{1}\right), p_{1} ; \ldots ; u\left(b_{n}\right), p_{n}\right]=\left[u_{1}, p_{1} ; \ldots ; u_{n}, p_{n}\right]$

dove la funzione $u$ assume valori reali. Si potrà anche scrivere 
$U=\left[u_{1}, p_{1} ; \ldots ; u_{n}, p_{n}\right]$ a indicare la variabile casuale $U$ che assume $i$ valori $u_{1}, \ldots, u_{n}$ con rispettive probabilità $p_{1}, \ldots, p_{n}$.

Un tipo comune di prospettiva probabilistica riguarda somme di denaro; la generica prospettiva di questo tipo sarà simbolizzata con $d=\left[x_{1}, p_{1} ; \ldots ; x_{n}, p_{n}\right]\left(x_{i} \leq x_{i+1}\right)$, essendo $x_{i}$ un numero reale, che esprime una somma di denaro (negativa, nulla o positiva); una tale prospettiva viene usualmente denominata come la corrispondente variabile casuale, con la lettera $X$. Si assume che la funzione utilità sia una funzione non decrescente della quantità di denaro cui si applica.

La funzione $u$ deve essere coerente con lo spazio delle preferenze fra le prospettive certe: se $b \leq k$, allora $u(b) \leq u(k)$. Ai valori della funzione $u$ non viene associato un significato "assoluto", e infatti tale funzione $u$ è definita a meno di trasformazioni lineari crescenti; in particolare, per semplificare i riferimenti l'utilità della prospettiva meno favorevole può essere posta uguale a 0 , e l'utilità della prospettiva più favorevole può essere posta uguale a 1 .

$\mathrm{Nel}$ confronto tra prospettive probabilistiche deve essere assicurata la condizione fondamentale di dominanza stocastica:

se $d=\left[u_{1}, p_{1} ; \ldots ; u_{n}, p_{n}\right]$ e $d^{\prime}=\left[u_{1}^{\prime}, p_{1} ; \ldots ; u_{n}^{\prime}, p_{n}\right]$

con $u_{i} \leq u_{i}^{\prime}$ per ogni $i$ (si dice che $d$ ' è maggiore stocasticamente di $d$ ), allora $u(d) \leqq u\left(d^{\prime}\right)$, e pertanto deve essere $u(d) \leq u\left(d^{\prime}\right)$.In altre parole, ogni proposta di funzione utilità deve soddisfare la condizione di dominanza stocastica. Per quanto detto, tale condizione deve essere assicurata anche nel caso particolare in cui le prospettive certe di riferimento siano somme di denaro.

Per quanto di impostazione totalmente diversa, i contributi classici fondamentali di D. Bernoulli (1738) e di von NeumannMorgenstern (1953) pervengono entrambi alla stessa valutazione sintetica della prospettiva probabilistica (2), consistente nella media aritmetica, o valore atteso, della variabile casuale $U$ :

$u(d)=\mathrm{E}(U)=\bar{u}=\sum u\left(b_{i}\right) p_{i}$

Ciò significa che, nella scelta fra due o più prospettive probabilistiche, l'individuo dovrebbe scegliere quella che massimizza l'utilità 
attesa (espressione usuale per indicare la media delle utilità) (cfr. Lindley, 1985, p. 59). Nel seguito si farà riferimento alla proposta di von Neumann-Morgenstern con la sigla N-M-S, comprendendovi il riferimento anche al successivo contributo di L.J. Savage (1954), in sostanziale accordo con tale impostazione.

La distinzione fondamentale fra le impostazioni di D. Bernoulli e N-M-S consiste nella definizione e determinazione delle utilità:

- per Bernoulli $u(b)$ è valutata in condizioni prive di rischio (utilità realistica),

- per N-M-S $u(b)$ discende come risultato del confronto di più lotterie; essa dipende dall'utilità realistica, ma anche dall'atteggiamento personale rispetto al rischio.

Il criterio dell'utilità attesa (o criterio EU=Expected Utility), per quanto ottenuto da von Neumann-Morgenstern come implicazione (teorema) di alcuni ragionevoli assiomi, e successivamente confermato da Savage (1954) col ricorso a un più ampio sistema assiomatico, è stato subito sottoposto a varie critiche, le quali hanno evidenziato che molti individui sicuramente razionali non si comportano - di fronte a specifici esempi di scelta fra due o più prospettive probabilistiche - secondo il criterio EU. La principale critica è venuta da Maurice Allais (1953), in un celebre articolo pubblicato nello stesso anno in cui vedeva la luce la terza edizione del volume di von Neumann-Morgenstern "Theory of games and economic behavior", comprendente la derivazione del criterio EU da un sistema assiomatico. L'articolo di Allais è noto in particolare per l'esposizione e il commento di un problema di scelta - cui si accennerà più oltre con maggiore dettaglio - generalmente noto come "paradosso di Allais"; ma ci preme soprattutto ricordare a questo punto l'avvertimento di Allais, più volte ripetuto nel corso dell'articolo: "l'erreur fondamentale de toute l'école américaine, c'est de négliger indirectement et inconsciemment, la dispersion des valeurs psychologiques" (1953, p. 544). Possiamo dire subito che la proposta di un nuovo criterio decisionale, contenuta nel presente articolo, prende le mosse proprio da questo suggerimento, e lo rende direttamente operativo mediante una funzione $u_{g}$ delle utilità (con associate le rispettive probabilità) che risulta dalla differenza fra due termini: l'utilità media (presente nel criterio EU) e un termine proporzionale a un comune indice di dispersione, cioè la deviazione media assoluta (ovvero media degli 
scarti assoluti delle singole utilità dalla loro media). Formalmente, tale criterio risulta:

$u_{g}(d)=\sum u\left(x_{i}\right) p_{i}-\lambda \sum\left|u\left(x_{i}\right)-\bar{u}\right| p_{i}=\bar{u}-\lambda \mathrm{MA}(U)$

dove si è indicato con $\mathrm{MA}(U)$ la deviazione media assoluta delle utilità, mentre $\lambda$ è un parametro, che può assumere valori positivi, nulli o negativi. Sui valori assumibili da $\lambda$ si tornerà in seguito; al momento basterà rilevare che valori positivi di $\lambda$ saranno indicativi di avversione al rischio (la situazione di gran lunga più comune), mentre valori negativi saranno associabili a individui favorevoli, o propensi, al rischio; per $\lambda=0$ si avrebbe l'applicazione del criterio EU: $u_{g}(d)=\bar{u}$. Si osserva che il criterio $u_{g}$ è invariante rispetto a trasformazioni lineari delle utilità, nel senso che, se $U=\left[u_{1}, p_{1} ; \ldots ; u_{n}, p_{n}\right]$ e $V=a+b U$, vale la seguente relazione:

$u_{g}(V)=u_{g}(a+b U)=a+b u_{g}(U)$.

Si avverte che il contributo di questo articolo si pone in diretta prosecuzione di una precedente proposta dello stesso autore (Frosini, 1997), ed è esso stesso una versione ridotta di un contributo assai più esteso (Frosini, 2010) disponibile nella serie E.P. del Dipartimento di Scienze statistiche dell'Università Cattolica di Milano (consultabile anche tramite internet). La disponibilità di questi contributi rende possibile restringere la successiva esposizione agli aspetti di maggiore novità contenuti nella memoria più recente, e nello stesso tempo permette di rinviare a tale articolo per gli sviluppi formali, che per ragioni di spazio non possono essere qui riportati in dettaglio.

\section{LA SPECIFICAZIONE OPERATIVA DELL'UTILITÀ DEL GIOCO}

Nella impostazione tradizionale, che risale a D. Bernoulli (1738), un individuo ha un comportamento neutrale rispetto al rischio se è indifferente fra il mantenimento di una data prospettiva (o gioco, o scommessa) $X=\left[x_{1}, p_{1} ; \ldots ; x_{n}, p_{n}\right]$, e lo scambio con la somma data dal valore atteso $\bar{x}=\sum x_{i} p_{i}$. Il comportamento di gran lunga più comune, cioè di avversità al rischio, implica invece che l'individuo è disposto ad 
accettare un "equivalente certo" ( $\mathrm{CE}=$ certainty equivalent) $x=\mathrm{CE}(d) \leq \bar{x}$ in cambio della scommessa. Un comportamento assai meno frequente, di propensione al rischio, porterebbe invece l'individuo a mantenere la scommessa a fronte dell'offerta del valore atteso monetario $\bar{x}$.

Questo "comportamento rivelato" (l'equivalente certo rispetto a una data prospettiva) appare come il risultato congiunto di (almeno) due fattori indipendenti, e cioè la curvatura della funzione utilità realistica e l'atteggiamento verso il rischio. Si può assumere pacificamente che la funzione utilità realistica sia (più o meno) concava - ovvero con utilità marginale decrescente - praticamente per tutti gli individui; tale funzione utilità interagisce con l'atteggiamento individuale rispetto al rischio.

Sia pure mantenendo la validità del precedente riferimento, che fa leva sul concetto di "equivalente certo", la presente impostazione basata sul criterio (4) - assume una definizione di neutralità, oppure avversione, oppure propensione al rischio, secondo il criterio $u_{g}$, basate sulle seguenti definizioni:

(a) un individuo è $g$-neutrale rispetto al rischio - per una data prospettiva $d-$ se $u_{g}(d)=\bar{u}$ (l'utilità della data prospettiva coincide con l'utilità attesa);

(b) un individuo è $g$-avverso al rischio se $u_{g}(d)<\bar{u}$;

(c) un individuo è $g$-propenso al rischio se $u_{g}(d)>\bar{u}$;

in altre parole, l'avversione o la propensione al rischio secondo il criterio $u_{g}$ dipendono dalla dispersione delle utilità intorno alla loro media.

L'impostazione proposta risulta già pienamente comprensibile nel caso più semplice di una prospettiva binaria $d=[0,1-p ; 1, p]$, che può essere riferita a un gioco che assicura la vincita di 1 se accade un evento $A$ con probabilità $p$, e il mantenimento dello status quo se l'evento $A$ non accade (ovvero se accade l'evento contrario). Il valore atteso (ovvero la media) di questa prospettiva è $p$. Per un esempio così semplice è possibile e utile rappresentare la funzione utilità realistica $u(x)$ - per 0 $\leq x \leq 1$, dove $x$ è una somma di denaro in una scala opportuna - e l'utilità del gioco $u_{g}(p)-$ per $0 \leq p \leq 1-$ sullo stesso grafico, all'interno di un quadrato di lato 1, come è fatto in Fig. 1.

In tale figura l'utilità realistica è rappresentata dalla funzione concava $u(x)=x^{0.7}$. Dati i possibili esiti 0 e 1 del gioco, la sua valutazione col criterio (4) dipende dalla probabilità $p$, e risulta 
$u_{g}(p)=p-2 \lambda p(1-p) \quad 0 \leq p \leq 1$

dato che l'utilità attesa è $p$, mentre la deviazione assoluta media è

$\mathrm{MA}=|0-p|(1-p)+|1-p| p=2 p(1-p)$

la curva corrispondente a $u_{g}(p)$ in Fig. 1 è calcolata per $\lambda=1 / 4$, ovvero $u_{g}(p)=p(1+p) / 2$.

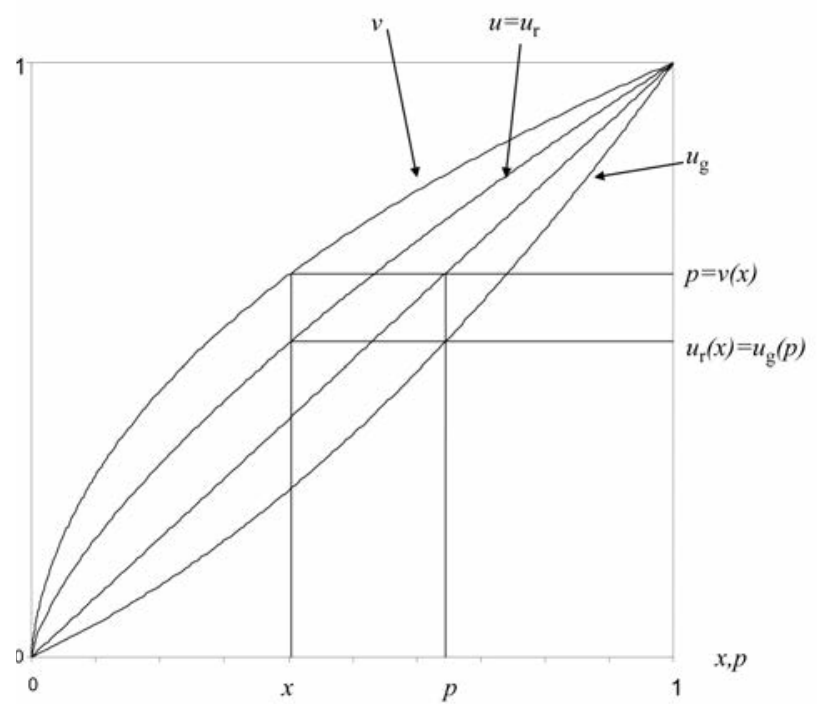

Fig. 1. Esempio di funzioni rilevanti per giochi binari nel caso di comportamento avverso al rischio. $x=$ risultato monetario del gioco, $p=$ probabilità di successo per il gioco

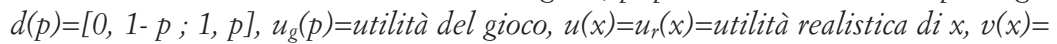
utilità N-M-S di x.

Per tale prospettiva binaria l'equivalente certo $x=\mathrm{CE}(d)$ dipende da $p$, e risulta dalla equazione

$u(x)=u_{g}(p) \rightarrow x(p)=u^{-1} \circ u_{g}(p) ;$

vale in generale

$u(x)=u_{g}(d) \rightarrow x(d)=u^{-1} \circ u_{g}(d)$. 
Continuando l'esempio numerico, vale $x(p)=u^{-1}(p)=[p(1+p) / 2]^{1 / 0.7}$

Si può inoltre osservare che, se si ricerca la funzione utilità $v(x)$ che fa corrispondere lo stesso equivalente certo sopra scritto alla valutazione del gioco ottenuta con l'impostazione N-M-S, si tratta di imporre l'equazione

$\bar{u}=p=u_{g}^{-1} \circ u(x)=v(x)$.

In tal modo si può controllare che la funzione utilità dell'impostazione N-M-S eguaglia la composizione $u_{g}^{-1} \circ u$; dato che $u_{g}$ è generalmente convessa, l'applicazione di $u_{g}^{-1}$ a $u$ esalta la concavità della funzione utilità realistica, come effettivamente avviene con l'esempio in Fig. 1. Ponendo $u=u(x)=x^{0.7}$, e tenuto conto che $u_{g}(p)=p(1+p) / 2$, dalla equazione $p^{2}+p-2 u=0$ si ricava la relazione, $\operatorname{per} p>0$ :

$p=-1 / 2+(1 / 4+2 u)^{0.5}$

da cui

$v(x)=u_{g}^{-1} \circ u(x)=-1 / 2+\left(1 / 4+2 x^{0.7}\right)^{0.5}$ per $0 \leq x, p \leq 1$.

\section{SODDISFACIMENTO DELLA DOMINANZA STOCASTICA \\ E DELLA CONDIZIONE DI INDIPENDENZA}

Nel caso delle prospettive binarie del tipo $d(p)=[0,1-p ; 1, p]$, per $0 \leq p \leq 1$, la dominanza stocastica di una prospettiva $d\left(p_{1}\right)$ rispetto a una prospettiva $d\left(p_{2}\right)$ è assicurata se $p_{1}>p_{2}$. Perché la funzione utilità $u_{g}$ soddisfi il criterio di dominanza stocastica, la funzione convessa (5) deve essere crescente da $p=0$ a $p=1$, cosa che accade effettivamente quando $0<|\lambda|<1 / 2$ (come si controlla facilmente). Si può "congetturare" che una media avversione al rischio sia rappresentata dal parametro $\lambda=1 / 4$, che implica la funzione utilità

$u_{g}(p)=p(1+p) / 2 \quad 0 \leq p \leq 1$. 
Allo stesso campo di variazione per $\lambda$ si arriva con una impostazione più generale, cioè con riferimento alla funzione $u_{g}(d)$ in formula (4), che si riferisce a un numero generico di prospettive certe (comprese nella prospettiva probabilistica); per la relativa dimostrazione si rinvia al par. 2.2 di Frosini (2010).

La validità della condizione di dominanza stocastica per la funzione $u_{g}(d)$ - nel caso generale - si può dimostrare in modo semplice, ancorché tedioso. Si imposta il calcolo di $u_{g}(d)$ per una generica prospettiva $d_{1}=\left[u_{1}, p_{1} ; \ldots ; u_{n}, p_{n}\right]$, e per una prospettiva variata $d_{2}$, nella quale un valore positivo $\Delta$ è trasferito da $p_{r}$ a $p_{s}$, essendo $u_{r}<u_{s}$; in tal modo la prospettiva $d_{2}$ risulta stocasticamente maggiore rispetto a $d_{1}$. Questo tipo di calcolo richiede verifiche distinte per i casi. (a) $u_{r}<u_{s} \leq$ $\bar{u}$, (b) $\bar{u} \leq u_{r}<u_{s}$, (c) $u_{r}<\bar{u}<u_{s}$, con vari sottocasi. In generale vale che, passando dalla prospettiva originaria $d_{1}$ alla prospettiva variata $d_{2}$, la funzione $u_{g}(d)$ aumenta in ogni caso se $|\lambda|<1 / 2$; come già accennato, valori negativi di $\lambda$ caratterizzano un individuo propenso (o favorevole) all'assunzione di rischio, mentre valori positivi di $\lambda$ si riferiscono al caso comune di individui avversi al rischio. Per la dimostrazione completa si rinvia a Frosini (2010), par. 2.2.

E' di notevole interesse, e in qualche modo inattesa, l'ulteriore proprietà di $u_{g}(d)$ di soddisfare la c.d. condizione di indipendenza. Tale condizione, ritenuta da molti studiosi una proprietà essenziale nell'ambito della teoria ortodossa dell'Utilità Attesa, afferma che, se la prospettiva $B$ è preferita alla prospettiva $A$, un miscuglio (probabilistico) di $B$ con qualunque altra prospettiva $C$ è sempre preferito al miscuglio fra $A$ e $C$. Si può dimostrare che tale proprietà vale in ogni caso se $|\lambda|<1 / 2$ (che è la stessa condizione stabilita più sopra per la validità della dominanza stocastica). Anche per questa dimostrazione si rinvia a Frosini (2010, par. 2.3); si osservi che il significato di tale condizione deve essere collegato, in questo caso, alla funzione utilità $u_{g}$, e non al criterio dell'utilità attesa (che costituisce un caso particolare del criterio $u_{g}$ ).

4. COMPORTAMENTI CONTRASTANTI FRA IL CRITERIO $u_{g}$ E IL CRITERIO DELL'UTILITÀ ATTESA

Un celebre esempio, proposto da Allais (1953) e generalmente denominato "paradosso di Allais", conduce per la grande maggioranza 
degli individui a un comportamento contrastante con l'applicazione del criterio dell'utilità attesa. Allais considera due situazioni che richiedono una decisione da parte di un individuo, cui sono proposti successivamente due giochi (o prospettive probabilistiche); la seguente esposizione utilizza cifre riferite a centinaia di migliaia di dollari (ad esempio, 5 significa 500.000 dollari).

Situazione 1 - Si può scegliere fra le prospettive:

$d_{1}$ : si ottiene 5 con certezza;

$d_{2}$ : status quo (cioè né vincite né perdite) con probabilità 0,$01 ; 5$ con probabilità 0,$89 ; 25$ con probabilità 0,10 .

Situazione 2 - Si può scegliere fra le prospettive:

$d_{3}$ : status quo con probabilità 0,$89 ; 5$ con probabilità 0,11 ;

$d_{4}$ : status quo con probabilità 0,$90 ; 25$ con probabilità 0,10 .

In grande maggioranza gli individui preferiscono $d_{1}$ a $d_{2}$, e $d_{4}$ a $d_{3}$; questo implica le seguenti diseguaglianze per qualunque funzione utilità $u$, se si adotta il criterio EU (utilità attesa):

$0.11 u(5)>0.1 u(25)+0.01 u(0)$

$0.11 u(5)<0.1 u(25)+0.01 u(0)$

che sono incompatibili. Altri controesempi, simili a questo ma che prevedono guadagni più moderati, sono stati proposti e controllati su campioni di individui da Kahneman e Tversky (1979).

Assumiamo ora che l'individuo $\mathrm{K}$, cui sono sottoposte le precedenti scelte, sia avverso al rischio, e adottiamo come criterio di scelta il criterio $u_{g}$ in formula (4). Siano $U_{1}, U_{2}, U_{3}, U_{4}$ le variabili casuali delle utilità associate rispettivamente ai giochi $d_{1}, d_{2}, d_{3}, d_{4}$. Consideriamo inoltre la tradizionale associazione delle utilità alla ricchezza posseduta, e assumiamo che le attuali disponibilità per $\mathrm{K}$ siano valutate 200.000 dollari. Con questi dati, dobbiamo valutare le compatibilità fra le seguenti diseguaglianze (già considerate da Frosini, 1997, p. 448):

$u(7)>0,1 u(27)+0,89 u(7)+0,01 u(2)-\lambda \mathrm{MA}\left(U_{2}\right)$

$0,1 u(27)+0,9 u(2)-\lambda \mathrm{MA}\left(U_{4}\right)>0,11 u(7)+0,89 u(2)-\lambda \mathrm{MA}\left(U_{3}\right)$;

ponendo $\delta_{i}=\mathrm{MA}\left(U_{i}\right)$, si controlla che le due diseguaglianze sono compatibili se $\delta_{2}+\delta_{3}-\delta_{4}>0$. 
Come possibile applicazione, consideriamo la seguente funzione utilità (da Lindley, 1985, p. 86):

$u(x)=1-\exp (-c x) \quad x>0 ; c>0$

se adottiamo inoltre il suggerimento di Lindley di associare il valore di utilità $1 / 2$ alla ricchezza esistente (200.000 dollari, per ipotesi), da cui $c=0,34657$, si ottengono i seguenti valori attesi e le seguenti deviazioni medie assolute:

$\bar{u}_{1}=0,91161, \delta_{1}=0 ; \bar{u}_{2}=0,91633, \delta_{2}=0,016717 ; \bar{u}_{3}=0,54528, \delta_{3}=0,080594 ;$ $\bar{u}_{4}=0,54999, \delta_{4}=0,089985$.

Con queste sostituzioni le precedenti diseguaglianze diventano:

$0.91161>0.91633-\lambda \times 0.016717$;

$0.54999-\lambda \times 0.089985>0.54528-\lambda \times 0.080594$.

Si può controllare che la diseguaglianza $\delta_{2}+\delta_{3}-\delta_{4}>0$ è soddisfatta. Inoltre, il campo di variazione dei valori $\lambda$ che permettono di soddisfare ambedue le diseguaglianze appare abbastanza ampio $(0,28199<\lambda<0,50201)$, e praticamente identico al campo di variazione dei valori ammissibili $0,28199<\lambda<0,5$.

Un altro paradosso, di natura più generale, presente nella teoria dell'utilità attesa, è il c.d. "paradosso dell'assioma di sostituzione"; esso è stato ampiamente studiato nel contributo fondamentale di Kahneman e Tversky (1979, pp. 266, 267, 282). In effetti, questi autori chiamano "assioma di sostituzione" quello che è usualmente indicato come "assioma - o condizione - di indipendenza", come fatto più sopra al par. 3. Tuttavia, Kahneman e Tversky propongono la seguente generalizzazione di vari esempi, di cui quello di Allais rimane l'archetipo: "The above problems illustrate common attitudes toward risk and chance that cannot be captured by the expected utility model. The results suggest the following empirical generalization concerning the manner in which the substitution axiom is violated. If $(y, p q)$ is equivalent to $(x, p)$, then $(y, p q r)$ is preferred to $(x, p r), 0<p, q, r<1$ " (p. 267). Avvertono subito gli autori - e dimostrano nel seguito del lavoro - che 
tale proprietà è incorporata nella teoria, chiamata "Prospect Theory", da essi proposta. Nel contributo di Frosini (2010, par. 7) viene dimostrato che l'adozione del criterio $u_{g}$ in formula (4) permette di verificare ugualmente la correttezza della empirical generalization proposta da Kahneman e Tversky.

Un'altra interessante applicazione, sempre proposta da Kahneman e Tversky $(1979$, pp. 269, 270, 285), il cui esito è contrastante con le implicazioni del criterio EU, riguarda la c.d. assicurazione probabilistica (probabilistic insurance). Il problema è così sinteticamente esposto nell'articolo richiamato (p. 270): "If at asset position $w$ one is just willing to pay a premium $y$ to insure against a probability $p$ of losing $x$, then one should definitely be willing to pay a smaller premium $r y$ to reduce the probability of losing $x$ from $p$ to $(1-r) p, 0<r<1$. Formally, if one is indifferent between $(w-x, p ; w, 1-p)$ and $(w-y)$, then one should prefer probabilistic insurance $(w-x,(1-r) p ; w-y, r p ; w-r y, 1-p)$ over regular insurance $(w-y)$ ". In realtà, il (ragionevole) comportamento degli individui è generalmente in senso opposto, cioè l'assicurazione probabilistica è ritenuta inferiore alla normale assicurazione. La dimostrazione di Kahneman e Tversky è confermata dalla applicazione del criterio $u_{g}$ in formula (4), come fatto da Frosini (2010, par. 6).

\section{SOluZione Al PARAdOSSO Di EllsberG}

Una diretta estensione della precedente impostazione, basata sul criterio $u_{\mathrm{g}}$, al caso delle decisioni sotto incertezza (cioè con limitata o nessuna conoscenza delle probabilità rilevanti per una data prospettiva), può essere semplicemente ottenuta; come immediata applicazione, il paradosso di Ellsberg (1961) può ricevere una convincente "spiegazione". La proposta presentata nel seguito è ripresa da Frosini (1997, pp. 453-454).

Allo scopo di definire un criterio immediatamente operativo, si ammetterà che l'individuo $\mathrm{K}$ sia in grado di stabilire, almeno come accettabile approssimazione, una distribuzione di probabilità sul vettore $\left(p_{1}, \ldots, p_{n}\right)$ con $\sum p_{i}=1$. Con questo riferimento, è possibile definire il comportamento (nella scelta fra più prospettive incerte) in presenza di una assegnazione imprecisa delle probabilità $p_{1}, \ldots, p_{n}$ agli stati di natura $s_{1}, \ldots, s_{n}$, come segue: 
(a) un individuo è $g$-neutrale rispetto al rischio se il problema di scelta con probabilità imprecise è equivalente al problema che si ottiene sostituendo ciascuna probabilità $p_{i}$ col suo valore atteso $\bar{p}_{i}=\mathrm{E}\left(p_{i}\right)(\mathrm{si}$ osservi che $\sum \bar{p}_{i}=1$ );

(b) un individuo è $g$-avverso al rischio se il problema di scelta è equivalente a un analogo problema con sostituzione di $p_{i}$ con un valore inferiore a $\mathrm{E}\left(p_{i}\right)$ quando la prospettiva certa collegata con lo stato $s_{i}$ è un guadagno, e con un valore superiore a $\mathrm{E}\left(p_{i}\right)$ quando la prospettiva collegata è una perdita o comunque nessun guadagno;

(c) un individuo è $g$-favorevole al rischio se il problema di scelta è equivalente a un analogo problema con sostituzione di $p_{i}$ con un valore superiore a $\mathrm{E}\left(p_{i}\right)$ quando la prospettiva certa collegata con lo stato $s_{i}$ è un guadagno, e con un valore inferiore a $\mathrm{E}\left(p_{i}\right)$ quando la prospettiva collegata è una perdita o comunque nessun guadagno.

Per ragioni di brevità, svilupperemo il criterio operativo solo nel caso comune di avversione al rischio, sia nel senso presentato nei precedenti paragrafi, sia nel senso della assegnazione di probabilità imprecise (ovvero della assegnazione di una distribuzione di probabilità in luogo di ogni $p_{i}$ ). Se le prospettive certe sono somme di denaro $x_{i}$, e usiamo il termine di correzione $\lambda \mathrm{MA}(U)$ applicato alle probabilità attese, l'espressione

$u_{g}(d)=\sum u\left(x_{i}\right) \bar{p}_{i^{-}} \lambda \sum\left|u\left(x_{i}\right)-\bar{u}\right| \bar{p}_{i}$

può essere usata quando il soggetto è avverso rispetto a prospettive rischiose $(\lambda>0)$ ed è neutrale rispetto alle probabilità imprecise. Se il soggetto è avverso al rischio anche rispetto alle probabilità imprecise, questa espressione può essere utilizzata egualmente, tuttavia sostituendo $\bar{p}_{i}$ con un $p_{i}^{*}$ più piccolo di $\bar{p}_{i}$ nel caso di prospettive positive, e con un $p_{i}^{*}$ più grande di $\bar{p}_{i}$ per prospettive negative o nulle. Anche in questo caso, per semplicità, potremmo usare un termine correttivo proporzionale alla deviazione media assoluta della variabile casuale $p_{i}$, ponendo

$p_{i}^{*}=\bar{p}_{i}-\gamma_{1} \mathrm{MA}\left(p_{i}\right), \quad \gamma_{1}>0$, per una prospettiva positiva, $p_{i}^{*}=\bar{p}_{i}+\gamma_{2} \mathrm{MA}\left(p_{i}\right), \quad \gamma_{2}>0$, per una prospettiva negativa o nulla.

Questa impostazione del trattamento delle probabilità imprecise 
permette di risolvere i paradossi che possono sorgere nella teoria classica dell'Utilità Attesa, quando non tutte le probabilità sono esattamente determinate, come nel caso del "paradosso di Ellsberg" (1961).

Paradosso di Ellsberg: E' noto che un'urna contiene 30 palline rosse, e 60 fra nere e gialle (in proporzioni ignote). Una pallina è estratta a caso dall'urna. Si considerano le seguenti possibilità di vincite:

Gioco 1: 100 dollari se è estratta una pallina rossa;

Gioco 2: 100 dollari se è estratta una pallina nera;

Gioco 3: 100 dollari se è estratta una pallina nera o gialla;

Gioco 4: 100 dollari se è estratta una pallina rossa o gialla.

E' stato verificato che la gran maggioranza degli individui preferiscono il gioco 1 al gioco 2, e il gioco 3 al gioco 4; tale comportamento implica la violazione del c.d. principio di certezza (sure-thing principle), e quindi della condizione di indipendenza (cfr. Frosini \& Giossi, 1994, par. 6).

Per semplicità, poniamo $0=$ status quo, e $u(0)=0$; ne consegue che le utilità delle possibili azioni possono essere scritte come segue (numerando le azioni $a_{1}-a_{4}$ come i corrispondenti giochi):

$u\left(a_{1}\right)=u(100)(1 / 3) ; u\left(a_{2}\right)=u(100) p_{2} ;$

$u\left(a_{3}\right)=u(100)(2 / 3) ; u\left(a_{4}\right)=u(100)\left(1 / 3+p_{3}\right)$.

Se assumiamo $\bar{p}_{2}=\bar{p}_{3}=1 / 3$ (come potrebbe ottenersi ad esempio da una distribuzione uniforme fra 0 e $2 / 3$ ), e corrispondentemente $p_{2}{ }^{*}=p_{3}{ }^{*}=1 / 3-\gamma(\operatorname{con} \gamma>0)$, le seguenti diseguaglianze sono immediatamente controllate:

$u\left(a_{2}\right)=u(100)(1 / 3-\gamma)<u\left(a_{1}\right) ; u\left(a_{4}\right)=u(100)(2 / 3-\gamma)<u\left(a_{3}\right) ;$

in tal modo il paradosso viene spiegato con la stessa applicazione del modello EU, applicato però alle probabilità $p_{i}^{*}$ (caso di $\lambda=0$ per il criterio $u_{g}$ ). Nel caso generale, la formula (4) può essere riscritta come segue:

$u_{g}(d)=\sum u\left(x_{i}\right) p_{i}^{*}-\lambda \mathrm{MA}^{*}=\bar{u}^{*}-\lambda \mathrm{MA}^{*}$

essendo MA* $=\sum\left|u\left(x_{i}\right)-\bar{u}^{* *}\right| p_{i}^{*}$. Ponendo per semplicità $u(100)=1$, otteniamo: 


$$
\begin{aligned}
& \bar{u}\left(a_{1}\right)=1 / 3 ; \bar{u}^{*}\left(a_{2}\right)=1 / 3-\gamma ; \bar{u}\left(a_{3}\right)=2 / 3 ; \bar{u}^{*}\left(a_{4}\right)=2 / 3-\gamma ; \\
& \operatorname{MA}\left(a_{1}\right)=|0-1 / 3|(2 / 3)+|1-1 / 3|(1 / 3)=4 / 9, \text { coincidente con } \operatorname{MA}\left(a_{3}\right) ; \\
& \operatorname{MA}^{*}\left(a_{2}\right)=|0-(1 / 3-\gamma)|(2 / 3+\gamma)+|1-(1 / 3-\gamma)|(1 / 3-\gamma)= \\
& =4 / 9-(2 / 3) \gamma-2 \gamma^{2} ; \\
& \operatorname{MA}^{*}\left(a_{4}\right)=|0-(2 / 3-\gamma)|(1 / 3+\gamma)+|1-(2 / 3-\gamma)|(2 / 3-\gamma)= \\
& =4 / 9+(2 / 3) \gamma-2 \gamma^{2} ; \\
& u_{g}\left(a_{1}\right)-u_{g}\left(a_{2}\right)=[1 / 3-(4 / 9) \lambda]-\left[(1 / 3-\gamma)-\lambda\left(4 / 9-(2 / 3) \gamma-2 \gamma^{2}\right]=\right. \\
& =\gamma(1-(2 / 3) \lambda-2 \lambda \gamma)
\end{aligned}
$$

con radici 0 e $1 /(2 \lambda)-1 / 3$. Dato che siamo interessati solo a valori $0 \leq \gamma$ $<1 / 3$, per $\lambda$ positivo (caso di soggetto avverso al rischio) la suddetta differenza è maggiore di 0 . Analogamente, la differenza

$$
\begin{aligned}
& u_{g}\left(a_{3}\right)-u_{g}\left(a_{4}\right)=[2 / 3-(4 / 9) \lambda]-\left[(2 / 3-\gamma)-\lambda\left(4 / 9+(2 / 3) \gamma-2 \gamma^{2}\right]=\right. \\
& =\gamma(1+(2 / 3) \lambda-2 \lambda \gamma),
\end{aligned}
$$

con radici 0 e $1 /(2 \lambda)+1 / 3$, è maggiore di 0 sotto le stesse condizioni per $\lambda$. Ne risulta che anche nel caso generale per il criterio $u_{g}$ il paradosso di Ellsberg è completamente spiegato.

\section{CONCORDANZA CON L'IMPOSTAZIONE Di QuigGin}

Una caratteristica interessante della funzione utilità qui considerata è che si tratta di una funzione utilità compatibile con una impostazione molto generale, e del tutto differente, che è diventata molto popolare negli ultimi anni, dovuta a J. Quiggin (1982-1993). Seguendo il suggerimento di Kahneman e Tversky (1979) di applicare opportune trasformazioni alle probabilità inserite nei criteri decisionali, Quiggin ha spostato l'attenzione dalle singole probabilità alle probabilità cumulate, mantenendo in questo modo alcune delle proprietà condivise dal modello EU; questa impostazione è usualmente riferita con l'acronimo RDEU (Rank-Dependent Expected Utility). L'utilità attesa propria del criterio EU viene sostituita da Quiggin con una "funzione di valutazione" (con simile apparenza):

$\mathrm{V}(\boldsymbol{x}, \boldsymbol{p})=\sum u\left(x_{i}\right) k_{i}(\boldsymbol{p})$

dove $k_{i}(\boldsymbol{p})=q\left(\sum_{r=1}^{i} p_{r}\right)-q\left(\sum_{r=1}^{i-1} p_{r}\right)$ 
Le probabilità cumulate originali vengono in tal modo trasformate per mezzo di una funzione di percezione $q$, che riflette la personale valutazione dell'individuo e il suo atteggiamento verso il rischio. Ponendo $u_{i}=u\left(x_{i}\right)$, e $P^{*}=\sum_{u_{i} \leq \bar{u}} p_{i}$, la deviazione media assoluta delle utilità può scriversi

$$
\begin{aligned}
\operatorname{MA}(U) & =\sum_{u_{i} \leq \bar{u}}\left(\bar{u}-u_{i}\right) p_{i}+\sum_{u_{i}>\bar{u}}\left(u_{i}-\bar{u}\right) p_{i} \\
& =\sum_{u_{i} \leq \bar{u}} 2 u_{i} p_{i}\left(P^{* *}-1\right)+\sum_{u_{i}>\bar{u}} 2 u_{i} p_{i} P^{* *} ;
\end{aligned}
$$

quindi, in applicazione del criterio $u_{g}$ in formula (4):

$$
\begin{aligned}
& u_{g}(d)=\bar{u}-\lambda \mathrm{MA}(U) \\
& =\sum_{u_{i} \leq \bar{u}} u_{i} p_{i}\left[1+2 \lambda\left(1-P^{*}\right)\right]+\sum_{u_{i}>\bar{u}} u_{i} p_{i}\left(1-2 \lambda P^{*}\right)
\end{aligned}
$$

che appare un caso speciale della funzione di valutazione (10); per un individuo avverso al rischio $(\lambda>0)$ le probabilità sono moltiplicate per $\left[1+2 \lambda\left(1-P^{*}\right)\right]>1$ for $u_{i} \leq \bar{u}$, e per $\left(1-2 \lambda P^{*}\right)<1$ for $u_{i}>\bar{u}$; in tal modo la trasformazione è concava, una proprietà compatibile con la supposta avversione al rischio (Quiggin 1993, pp. 59, 78). Si controlla immediatamente che le probabilità trasformate sommano a uno:

$=\sum_{u_{i} \leq \bar{u}} p_{i}\left[1+2 \lambda\left(1-P^{*}\right)\right]+\sum_{u_{i}>\bar{u}} p_{i}\left(1-2 \lambda P^{*}\right)=1$.

Inoltre, osserviamo che il coefficiente di $p_{i}$ nel secondo termine di formula (11) è positivo solo se $\lambda<1 / 2 P^{*}$; dato che sup $P^{*}=1$, troviamo nuovamente che $\lambda$ non può superare $1 / 2$.

Come esempio, per la prospettiva

$d=[0,0.05 ; 0.5,0.25 ; 0.8,0.60 ; 1,0.10]$

otteniamo $\bar{u}=0,705, P^{*}=0,3$, e i seguenti coefficienti (cfr. formule (10) e (11)):

$k_{1}=0,05(1+2 \times 0,4 \times 0,7)=0,078$

e analogamente: $k_{2}=0,25 \times 1,56=0,39, k_{3}=0,6 \times 0,76=0,456, k_{4}=0,1 \times 0,76=$ 
0,076 (si osservi che $k_{1}+\ldots+k_{4}=1$ ); ne risultano i valori $q\left(p_{1}\right)=0,078$, $q\left(p_{1}+p_{2}\right)=0,468, q\left(p_{1}+p_{2}+p_{3}\right)=0,924, q\left(p_{1}+\ldots+p_{4}\right)=1$. La concavità della trasformazione è chiaramente apprezzabile dalla Fig. 2.

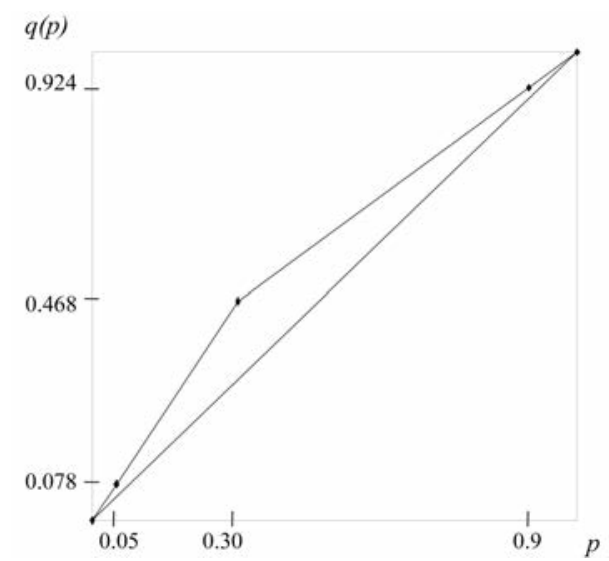

Fig. 2. Funzione di trasformazione $\mathrm{q}(\mathrm{p})$ per l'esempio nel testo.

\section{ESPERIMENTO}

E' stato realizzato un esperimento, di tipo usuale in questo tipo di verifiche, chiedendo a venti persone (alcuni colleghi e alcuni studenti di Economia) di rispondere a dieci domande di un apposito questionario, in cui venivano proposti giochi (ovvero prospettive probabilistiche), che prevedevano soltanto guadagni (limitati) o il mantenimento dello status quo. La procedura concretamente seguita è stata ripresa, quasi esattamente, da quella analoga realizzata da Tversky e Kahneman (1992, pp. 306-307).

Lo scopo dell'esperimento - anche tenuto conto del piccolo numero di partecipanti - non era rivolto al controllo empirico del comportamento effettivo degli individui, ma solo alla verifica operativa del criterio $u_{g}$. I risultati dell'esperimento, e la verifica numerica dei parametri del modello, sono stati del tutto soddisfacenti. L'esposizione dettagliata del questionario, e delle stime numeriche, sono consultabili al par. 10 di Frosini (2010). 


\section{RIFERIMENTI BIBLIOGRAFICI}

M. Allais 1953, Le comportement de l'homme rationnel devant le risque: Critique des postulats et axioms de l'école américaine, Econometrica, 21 (1953), 503-546.

D. Bernoulli 1738, Exposition of a new theory on the measurement of risk (traduzione dal latino), Econometrica, 22 (1954), 23-36.

D. Ellsberg 1961, Risk, ambiguity, and the Savage axioms, Quarterly Journal of Economics, 75 (1961), 643-669.

B.V. Frosini \& L. Giossi 1994, La comparazione tra prospettive incerte: modelli teorici e comportamento effettivo, Istituto di Statistica, Università cattolica del Sacro Cuore, Serie E.P. n. 63.

B.V. Frosini 1997, The evaluation of risk attitudes: A new proposal, Statistica Applicata, 9 (1997), 435-458.

B.V. Frosini 2010, Realistic utility versus game utility:A proposal for dealing with the spread of uncertain prospects, Dipartimento di Scienze statistiche, Università Cattolica del Sacro Cuore, Serie E.P. n. 140.

KD. Kahneman \& A. Tversky 1979, Prospect theory: An analysis of decision under risk, Econometrica, 47 (1979), 263-291.

D.V. Lindley 1985, Making Decisions (2nd Edition), London, Wiley, 1985.

J. Quiggin 1982, A theory of anticipated utility, Journal of Economic Behavior and Organization, 3 (1982), 323-343.

J. Quiggin 1985, Anticipated utility, subjectively weighted utility and the Allais paradox, Organizational Behavior and Human Performance, 35 (1985), 94-101.

J. Quiggin 1993, Generalized Expected Utility Theory: The Rank-Dependent Model, Boston, Kluwer, 1993.

L.J. Savage 1954, The Foundations of Statistics, New York, Wiley, 1954.

A. Tversky \& D. Kahneman 1992, Advances in prospect theory: Cumulative representation of uncertainty, Journal of Risk and Uncertainty, 5 (1992), 297-323.

J. von Neumann \& O. Morgenstern 1944-1953, Theory of Games and Economic Behavior (3rd Edition), Princeton, Princeton University Press, 1944/1953. 